\title{
Multicolor Solitons for Biosensors
}

\section{Tamee $\mathbf{K}^{1}$, Visessamit $\mathbf{J}^{2}$ and Yupapin $\mathbf{P P}^{2,3 *}$}

${ }^{1}$ Department of Computer Science and Information Technology, Faculty of Science, Naresuan University, Pitsanulok 65000, Thailand

${ }^{2}$ Advanced Studies Center, Department of Physics, Faculty of Science, King Mongkut's Institute of Technology Ladkrabang, Bangkok (KMITL), Thailand

${ }^{3}$ South East Asian Theoretical Physics Association (SEATPA), Singapore

Optical soliton has been recognized as a good candidate for long distance optical communication and long lasting propagation in optical waveguide, where the dominant properties are high output intensity, low loss and small dispersion value within the propagation media, the concept of repeater less is also the another good advantage. However, the narrow band condition that gives soliton application limitation is the pumping device wavelength, where the pumping material used is the specific material known as an erbium doped waveguide (fiber), in which the pumping energy at the center wavelength is 1.55 micrometer. Therefore searching for multi-wavelength solitons with new pumping technique has been the challenge. Till date, many researchers have investigated and reported the possibility of multi-wavelength soliton generation, where most of them proposed the soliton generations in many aspects [1-5], in which the color solitons [6-8], however, the previous problems remain in practical applications.

Recently, Yupapin et al have reported the use of an interesting system and results that can be used to form multicolor solitons, where the color solitons are now easily generated and controlled by using the Gaussian input pulse (common laser pulse) propagating within typical modified add-drop filter [9-11], in which two nonlinear devices are used incorporating the center ring known as a PANDA ring circuit. In operation, light from a common laser source with wide range of center wavelength is input into the system as shown in (Figure 1). The nonlinear behavior of light propagation within the system is occurred by the coupling effects from the two nonlinear side rings, in which the four-wave mixing of light can be introduced within the system due to the superposition of the chaotic signals, which is generated by the two nonlinear side rings. Finally, the resonant situation of some wavelengths can be introduced and pumped the propagation modes and seen at the system output port, where in this case the multi-wavelength output signals are obtained, in which the two soliton properties (i) self-phase and (ii) cross phase modulation without dispersion remain. To change the multiwavelength or color soliton bands, the use of the control port is required by input the moderated signal via the add port, in which multicolor solitons can be generated and controlled for various applications.

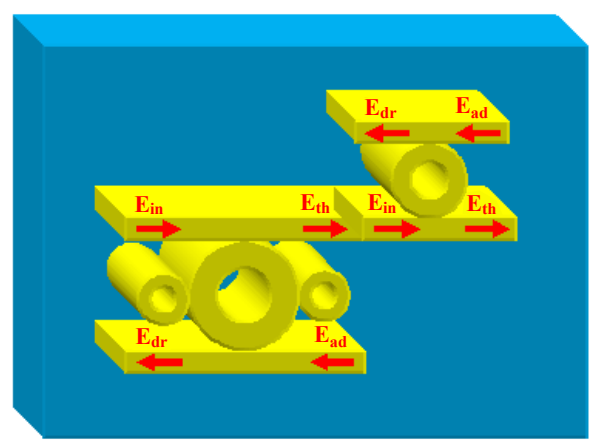

Figure 1: Color soliton generation system, where $E_{\text {in }}$ :input port field; $E_{d r}$ : drop port field; $E_{a d}$ : add port field; $E_{t h}$ : through port field, where more add-drop filters can be included for long distance use.
In simulation, a common laser (i.e. laser pointer) is exploited as a laser source. The optical power is the same as a common laser peak power, which is normally at $3.0 \mathrm{~mW}$, however, the normalized output power is required and plotted. By using the system in (Figure 1), the simulation results are obtained by using the practical parameters, the

MATLAB program is used to obtain the results. The common laser pulse can be changed to be a soliton pulse by the resonant pumping power via the two side rings, which is occurred and seen via the add-drop filter output $\left(\mathrm{E}_{\mathrm{th}}\right)$. In this simulation, the center ring radius of $20 \mu \mathrm{m}$ is supposed to be the input main ring circuit as shown in (Figure 1), the two nonlinear side ring radii are $5 \mu \mathrm{m}$. The center ring and nonlinear materials are $\mathrm{SiO}_{2}$ and $\mathrm{In} \mathrm{GaAsP} / \mathrm{InP}$ respectively. The waveguide attenuation coefficients $(\alpha)$ is $0.2 \mathrm{~dB} / \mathrm{mm}$ [12]. The output transfer function is obtained by using the signal flow graph method [13]. The resonant number of the main ring and side rings (N: NR: NL) are chosen as 9:6:6. The outer coupling factors $\kappa_{1}$ and $\kappa_{2}$ of PANDA ring resonator are fixed as 0.65 and the inner coupling factors $\left(\kappa_{R}\right.$ and $\kappa_{\mathrm{L}}$ ) are set as 0.35 . A round-trip time of the PANDA circuit at the

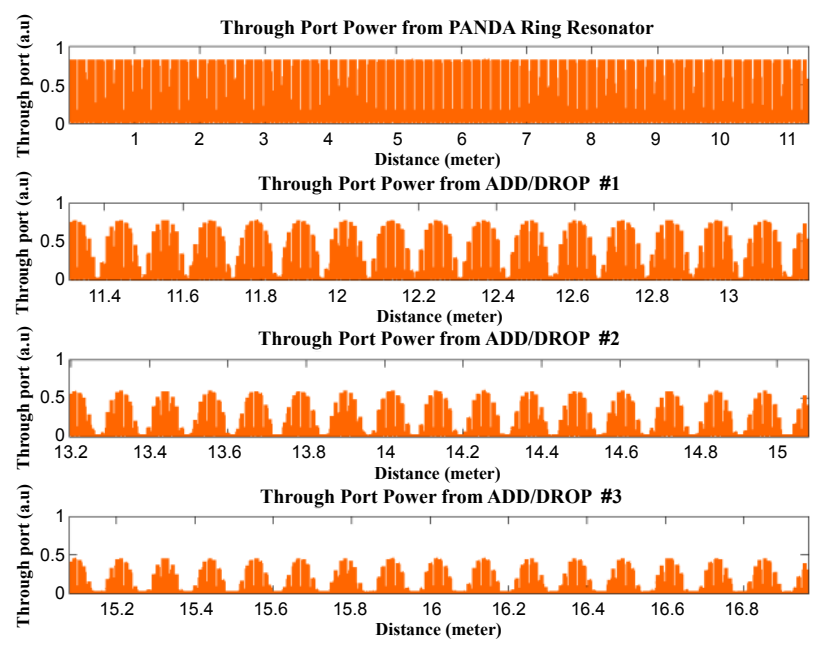

Figure 2: Results of color soliton propagation in the system with wavelength center at 0.5775 micrometer, where the normalized power has slightly changed from 0.8 to 0.5 with distance of 16.6 meter.

*Corresponding author: Yupapin PP, Advanced Studies Center, Department of Physics, Faculty of Science, King Mongkut's Institute of Technology Ladkrabang, Bangkok (KMITL), Thailand, 3South East Asian Theoretical Physics Association (SEATPA), Singapore, E-mail: kypreech@kmitl.ac.th

Received July 20, 2013; Accepted July 21, 2013; Published July 22, 2013

Citation: Tamee K, Visessamit J, Yupapin PP (2013) Multicolor Solitons for Biosensors. J Biosens Bioelectron 4: e122. doi:10.4172/2155-6210.1000e122

Copyright: @ 2013 Tamee K, et al. This is an open-access article distributed under the terms of the Creative Commons Attribution License, which permits unrestricted use, distribution, and reproduction in any medium, provided the original author and source are credited. 


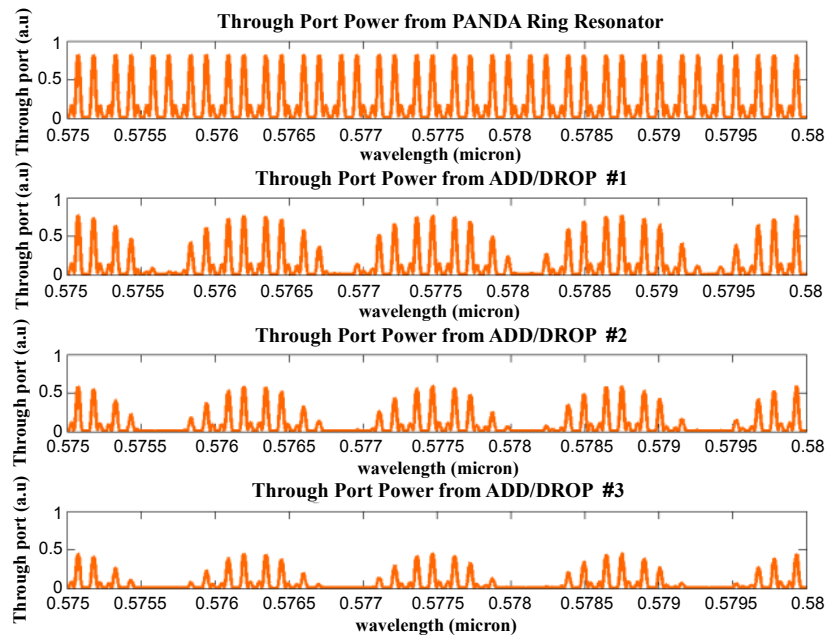

Figure 3: Color soliton output signals, the center wavelength is 0.5775 micron, in which the multicolor solitons (different wavelengths) is obtained by using the system in Figure 1.

resonant is $4.35 \mathrm{ps}$. The obtained power (arbitrary unit) from through port of PANDA ring, first add-drop, second add-drop and third adddrop are $82.19 \%, 76.81 \%, 59.00 \%$ and $45.32 \%$ comparing with the input port of the PANDA ring circuit, respectively. The simulation results of color soliton are obtained, where the obtained soliton power with propagation distance is as shown in (Figure 2), in which the concept of soliton is occurred because the soliton power attenuation is slightly seen along the propagation distance, which the soliton power can be recovered by two soliton behaviors known as self-phase and cross phase modulations. The soliton outputs with different wavelengths are obtained and shown in (Figure 3), where in general the multicolor solitons can be easily obtained by using the same method. The other waveguide parameters in this simulation are $\mathrm{A}_{\text {eff }}=0.25 \mu \mathrm{m}^{2}, \mathrm{n}_{\text {eff }}=3.14$, $\mathrm{n}_{2}=1.3 \times 10^{-13} \mathrm{~cm}^{2} / \mathrm{W}$.

Furthermore, results of the through port outputs of (Figure 1) can be used for longer propagation distance in the transmission system, in which the required devices are add-drop filters. The soliton outputs are also obtained as shown in (Figures 2 and 3), the through port results from the longer distances are obtained by using the Add and Drop ports $\# 2$ and \#3, which is confirmed that the soliton behaviors remain, where the optical power has slightly changed from 0.8 to 0.5 at the center wavelengths.

In applications, multicolor solitons have shown the promising applications, especially, for long distance propagation within the light media, where (i) they offer a truly unique laboratory for soliton phenomena: multicolor solitons exist in the spatial and the temporal domains; they exist in guided and in bulk media; they exist in any physical dimensions and thus have the potential to form threedimensional light bullets; they exist in continuous as well as in discrete physical settings; and they are the realization of a universal phenomenon in nature, namely, the nonlinear parametric mixing of waves, where (ii) multicolor solitons offer clean, robust, stabilized, particle like pulses. These beams could play key roles in future passive or active, single pass or cavity, multi-frequency photonic devices for which the price of operation at high peak power is offset by the unique features of the soliton light spots. Moreover, the use of soliton communication and security can be seen in the transmission line, where the new device soliton repeater (recovery) can be realized, which is useful for soliton recovery after drop signal being recovered and used in the transmission network.

In conclusion, the use of multicolor solitons can be implemented by small scale optical devices and systems, in which the dominant soliton behaviors such as long lasting propagation time with various wavelengths can be useful for biosensor and bioelectronics applications, moreover, the multicolor solitons can be realized within the small scale optical systems, where in this case the propagation length of 16.6 meters at the center wavelength of 0.58 micrometer is confirmed.

We would like to thank the King Mongkut's Institute of Technology Ladkrabang (KMITL), Bangkok, Thailand for providing the research facilities.

\section{References}

1. Kato M (2006) Wavelength-tunable color Raman soliton generation using an ellipse polarized pump pulse and highly birefringent optical fibers. Lightwave Technol 24: 805-809.

2. Assanto G, Smyth NF (2013) Comment on "Solitons in highly nonlocal neumatic liquid crystals: Variational approach. Phys Rev A 87: 047801-2.

3. Kato M, Mori Y (2006) Determination of actual interaction length for selffrequency shift of Raman solitons and their independence of pump intensities. Photon Techn Lett 18: 1386-1388.

4. Torner L, Barthelemy A (2003) Quadratic solitons: recent developments. IEEE Quant Electron 39: 22-30.

5. Kartashov YV, Vysloukh VA, Torner L (2013) Solitons in spiraling Vogel lattices Opt Lett 38: 190-192.

6. Kartashov YV, Torner L,Vysloukh VA (2004) Multicolor lattice soliton. Opt Lett 29: $1117-1119$

7. Lundquist PB, Andersen DR, Kivshar YS (1998) Multicolor solitons due to fourwave mixing. Phys Rev E 57: 3551-3555.

8. Huang C, Li C, Dong L (2013) Stabilization of multimode solitons in mixed linear-nonlinear lattices with a PT symmetry. Opt Express 21: 3917-3925.

9. Yupapin PP (2013) Nonlinear coupling effects of Waves in a Panda Ring Science Discovery 1: 1-5

10. Yupapin PP, Nimcome S (2013) Conjugate mirror by a PANDA ring circuit. Science Innovation 1: 1-4.

11. Yupapin PP, Thammawongsa, Jalil MA, Ali J (2013) Whispering gallery mode of wave in small scale optical device: The challenges. Scientific Reports NPG 3 Accepted.

12. Prabhu AM, Tsay A, Han Z, Van V (2010) Extreme miniaturization of silicon add drop microring filters for VLSI photonics applications. IEEE Photon 2: 436-444.

13. Bahadoran M, Ali J, Yupapin PP (2013) Ultrafast all-optical switching using signal flow graph for PANDA resonator. Appl Opt 52: 2866-2873. 